\title{
A Cognitive Account of Mandarin Chinese Jin
}

\author{
Li Larry Hong-lin \\ General Education Center, National Taiwan University of Arts, Taiwan \\ *Corresponding Author: larryli@ntua.edu.tw
}

Copyright (C) 2014 Horizon Research Publishing All rights reserved.

\begin{abstract}
In this study, we explore the polysemy of Jin. It is found that the polysemous network of Jin can be divided into two groups. The first sense group is composed of three senses. The sense 'to proceed' is the prototype of the first group. As in the LIFE IS A JOURNEY metaphor, progress in terms of time can be viewed as the distance traveled, and hence another sense arises. As for another sense, the GOOD IS FRONTWARD metaphor accounts for why Jin stands for good. The second sense group comprises five senses. Jinlai 'to enter a bounded space' is the central instance of the second group. The containment metaphor, which conceptualizes not only physical objects but also non-physical phenomena including an area, a stomach for digesting, eyesight, and wealth as containers, provides a basis for why Jin are tied to the other three senses of the second sense group, all of which describe an entity moving into the inner space. The findings of this study indicate that the discrepancy between language and mental process is not one of kind; in addition, meaning is built on the conceptual structure formed by the interaction with the outer world rather than objective reality.
\end{abstract}

Keywords Polysemy, Metaphor, Cognition, Prototypicality

\section{Introduction}

Human beings categorize things, based on the features that things share. If language is shaped by cognition, then the polysemy of a lexical item is by definition a product of human categorization. That is, lexical polysemy arises out of the effects of human categorization. This research, from a cognitive viewpoint, explores how the polysemy of Mandarin Chinese Jin emerges.

This research is motivated by the fact that Jin is associated with multiplex senses. For instance, in qianjin 'to proceed', Jin describes something moving horizontally in space. However, in jinzhan 'to develop as time goes by', Jin turns to describe the progress of events or actions. Still in jinlai 'to go into a space', Jin portrays something moving into a contained space. From these examples, we suggest that the polysemous nature of Jin is worth investigating.
This research adopts Lakoff and Johnson[1] to define the polysemy of Jin as involving a semantic network. This semantic network is composed of distinct sense groups, which can be further analyzed into minor senses. Prototypicality exists not only among sense groups, but also among the minor senses in each group. The central prototype in this polysemous category of Jin can be described in terms of the path schema. For the other related senses, they can be profiled by adding some extra information to the basic path schema or by metaphorical transformations.

In this study, we list all the senses tied to Jin. Subsequently, we identify the prototypical meaning of the polysemy. Then, all the other meanings are ranked, depending on their relevance to the central origin.

This paper is organized as follows. Section $\mathbf{2}$ discusses the theories needed in this research. What follows is section $\mathbf{3}$, which lists the multiplex senses of Jin and identifies the prototypical sense. Section 4 tackles the first sense group, and section 5 takes care of the second sense group. Lastly, section $\mathbf{6}$ is the conclusion.

\section{Theoretical Foundation}

In this study, the idea of radial categories is introduced. In addition, the path schema is employed as to account for why the polysemy of Jin can be divided into two major sense groups. Also, we present relevant metaphorical transformations needed to capture the polysemy of Jin.

\subsection{Radial Categories}

Regarding prototypicality, it refers to the engenderment of multiplex meanings of a polysemous lexeme stemming from a central prototype [2]. These related but distinct meanings can be classified into major sense groups, which can be categorized into minor senses. Furthermore, prototypicality not only exists between major sense groups, but also extends between minor senses in each major sense group. In sum, these senses and sense groups constitute a semantic network, which is tagged "radial category".

\subsection{The Path Schema}

The path schema, based on our experience of either 
moving in the world or sensing the movements of other entities, is employed to organize thoughts across a range of abstract domains [3]. In the case of journey, our experiences tell us that a journey always has a beginning, an end, and different places on the way. Also, a movement may include a route, just like the flight of an entity traveling through the air. Founded on our understanding of what a movement is like, the path schema is a template composed of a starting point, an end point, a projected path, a landmark and a moving object.

Cognitively, extra information can be added to the path schema, which leads to a modification of the basic schema [4]: for instance, the landmark can be shifted from two-dimensional to three-dimensional, changing the point-to-point movement of the trajector into the space-to-space one.

\subsection{Conceptual Metaphors}

Cognitive linguists explain that metaphors are means whereby abstract ideas can be conceptualized in terms of the concrete $[5,6]$. In other words, metaphors are mappings to establish correspondences between the entity in the concrete (source) domain and abstract (target) domain.

\section{Life Is A Journey}

In the metaphor LIFE IS A JOURNEY, time is compared to a stationary landmark, and all the events, activities, or actions conducted by people are profiled as the moving object traversing the landmark [7]. Therefore, the progress of the event and action can be viewed as the distance traveled.

\section{Good Is Frontward}

The metaphor GOOD IS FRONTWARD is grounded on the ME-FIRST orientation [8]. In this metaphorical model, both FRONT and GOOD are oriented toward the canonical person, while both BACK and BAD are oriented away from the canonical person. It follows that, in cognitive terms, FRONT and BACK, which are spatial domain, are respectively linked to GOOD and $\mathrm{BAD}$, which are abstract domain.

\section{The Containment Metaphor}

According to the model of the containment metaphor, human beings view physical entities, which are bounded by surfaces, as containers. Even if the entity is without any physical boundary, our minds mark off territory so that it has a confined surface. Moreover, many non-physical phenomena are also conceptualized as containers, which are bounded and have an in-out orientation. The container metaphor is crucial for our exploration of the sense extension of Jin.

\section{Sense Extension of $\boldsymbol{J i n}$}

Before identifying the prototypical meaning of Jin, we list all the senses pertaining to Jin. Among all the senses, the origin of meaning derivation is identified. Subsequently, by looking into the family resemblance, we examine how the senses of Jin can further categorized.

\subsection{Multiplex Senses of Jin}

In order to collect the senses concerning Jin, we consult two dictionaries, Ciyuan[9] and Guoyuribao Cidian[10]. We present the results as follows.

Sense 1: to move forward in space, e.g. qianjin 'to proceed' Sense 2: to progress in terms of time, e.g. jinzhan 'to develop as time goes by'

Sense 3: to be upgraded to a higher state or position, e.g. jinjie 'to advance'

Sense 4: to enter a bounded space, e.g. jinlai 'to go into a space'

Sense 5: to enter an area, e.g. jinkou 'to import the goods'

Sense 6: to eat something, e.g. jinshi 'to have food'

Sense 7: to present something, e.g. jingong 'to pay tribute to the suzerain'

Sense 8: to have a money income, e.g. jinzhang 'to receive money'

Given the senses, we proceed to analyze the origin of sense expansion of Jin.

\subsection{Prototypical Instance and Major Senses}

The prototypical instance of the polysemous network is the one involving the concrete domain [11]. Our analysis indicates that sense 1 and sense 4 of $\operatorname{Jin}$ are related to the spatial domain, describing objects moving in space. From a cognitive point of view, the spatial domain serves as the basis, whereby the abstract idea is transferred into the concrete concept and made more accessible [12]. Furthermore, when sense 1 and sense 4 are contrasted, it is found that sense 1, portraying an object moving in space, perfectly fits for the path schema. On the contrary, sense 4, describing an object moving into an enclosed space, can be compared to a schematic model by adding extra information to the basic path. The added information is that the landmark is changed from two-dimensional scene to three-dimensional space. In a word, sense 1 of Jin is the prototypical meaning of the whole category and sense 4 , the second major sense, is derived from sense 1 .

Having identifying the major senses in the category, we move on to group the other senses, depending on which one of them is extended from sense 1 and which one of them is derived from sense 4 . Sense 1 and the senses extended from sense 1 are the first major sense group and sense 4 and the senses originating from sense 4 are the second. The sense derivation in the two major groups of senses is illustrated as follows.

\section{The First Sense Group}

As we have elucidated, sense 1 of Jin is the prototypical meaning of not only the whole network, but also the first major sense group. In what follows, we tackle how sense 1 
engenders distinct but related senses via metaphor.

\subsection{Sense 1: Moving in Space}

In its first sense, Jin indicates an object moving forward in space. As we have clarified, sense 1 of Jin can be described by means of the basic path schema. Expressions illustrating this sense of Jin are listed as follows.

(1) a. qianjin 'to proceed'

b. jingong 'to proceed and attack the target'

c. jinbing 'to dispatch the troops closer to the enemy'

In (1a), (1b), and (1c), Jin portrays entities, including human beings, moving ahead in space. This sense, concerning a physical movement occurring in space, is concrete and fundamental in human cognition. Through mental processes, such as metaphorical mapping, sense 1 of Jin develops more distinct senses as illustrated in the rest of this section.

\subsection{Sense 2: Progressing in Time}

Sense 2 of Jin is extended from sense 1 through the metaphor LIFE IS A JOURNEY. In this metaphorical transferring, time is conceptualized as the stationary landmark, where people leading their lives are seen as going along this landmark. In other words, the progress of events, activities, and actions are viewed as objects traversing the land. Thus, sense 2 of Jin emerges. Expressions exemplifying this sense of Jin are illustrated as follows.

(2) a. jinzhan 'to develop as time goes by'

b. jindu 'the progression with respect to time'

c. jincheng 'the advancement of a plan as scheduled by time'

As in (2a), (2b), and (2c), Jin is related to the non-physical domain, describing abstract ideas. In what follows, the derivation of a sense regarding a state will be mentioned.

\subsection{Sense 3: Being Promoted to a Higher Status}

Sense 3 of Jin, coding a shift to a better state, stems from sense 1 by virtue of the GOOD IS FRONTWARD metaphor. This analogical process is grounded on the ME-FIRST orientation. That is, the canonical person tends to associate the good, the abstract domain, with the front, the concrete concept. Based on this line of reasoning, advancing to a better state is linked to moving forward in space. That is why sense 3 of Jin arises through extension from sense 1. Three examples demonstrating sense 3 are given below.

(3) a. jinjie 'to advance'

b. jinsheng 'to be promoted'

c. jinbu 'to improve'

In these three examples, (3a), (3b), and (3c), Jin is connected to a state including a status or a degree, which pertains to a more abstract realm.

\section{The Second Sense Group}

Sense 4 of Jin is the second major sense derived by adding extra information to the path schema, which serves as the prototype for many minor senses. We account for the meaning extension as follows.

\subsection{Sense 4: Going to a Bounded Space}

Sense 4 of Jin is associated to the landmark in the path schema undergoing a shift from two-dimensional scene to three-dimensional space. That is to say, the trajector enters the three-dimensional land, an enclosed space. Examples are shown as follows.

(4) a. jinlai 'to go into a space'

b.jinru 'to enter a room'

c. jinqu 'to make the way into a room'

In (4a), (4b), and (4c), Jin describes an object moving from an open space into a closed one.

\subsection{Sense 5: Entering an Area}

According to the containment metaphor model, an area without a boundary is conceptualized as a space having a bordered boundary like a container. Therefore, in an analogical manner, Jin codes an entity entering an area. Witness the following expressions.

(5) a. jinkou 'to import the goods'

b. jinchiang 'to be in an enclosed area'

c. jinjing 'to enter the capital'

As in example (5a), (5b), and (5c), sense 5 of Jin can be profiled as an entity going to a piece of land, on which our mind imposes a boundary.

\subsection{Sense 6: Having Food}

Sense 6 of Jin is built on sense 4 by imagining the stomach as a container. Precisely, in our cognition, the food being eaten is understood as the object entering a container. The expressions listed below can illustrate how sense 6 arises through the mental process being mentioned.

(6) a. jinshi 'to have food'

b. jincan 'to have a meal'

c. jinbu 'to take tonic'

(6a), (6b), and (6c) reveal that Jin portrays the act of eating.

\subsection{Sense 7: Presenting Something}

Sense 7 of Jin comes from sense 4, owing to the metaphorical mapping that the visual field is understood as a container. In other words, to present something is to have an object in a container. Following this line of reasoning, Jin produces the sense of presenting something as shown in the examples below.

(7) a. jingong 'to pay tribute to the suzerain'

b. jinxian 'to offer a gift to the superior'

c. jincheng 'to hand in something to the supervisor'

In the expressions of (7a), (7b), and (7c), Jin imparts the sense of describing an object being presented. 


\subsection{Sense 8: Having a Money Income}

Sense 8 of Jin appears by virtue of the metaphor that wealth is a container. In this conceptualization, getting money is compared to putting an object into a container. The following examples demonstrate the derivation of sense 8 .

(8) a. jinzhang 'to receive money'

b. jinkuan 'to receive money in the bank account'

c. jinxiang 'to get money'

In this sense, Jin, as in (8a), (8b), and (8c), depicts the act of getting money.

\section{Conclusion}

In our conclusion, our analysis of Jin is summarized. Subsequently, we draw the implication of our investigation. The polysemous network of Jin can be divided into two groups: the first group is composed of senses 1 to 3 ; the second senses 4 to 8 . According to the path schema, these two groups of senses pertain to a trajector moving along the path. As extra information can be added about the landmark, the landmark in the first sense group concerns a two-dimensional scene while it is changed into a three-dimensional field in the second sense group.

Sense 1 of Jin, 'to proceed', is the prototype of the first group. As in the LIFE IS A JOURNEY metaphor, progress in terms of time can be viewed as the distance traveled, and hence sense 2 arises. As for sense 3, the GOOD IS FRONTWARD metaphor accounts for why Jin stands for good.

Sense 4 of Jin 'to enter a bounded space' is the central instance of the second group. The containment metaphor, which conceptualizes not only physical objects, but also non-physical phenomena including an area, a stomach for digesting, eyesight, and wealth as containers, provides a basis for why Jin are tied to sense 5, 6, 7, and 8, describing an entity moving into the inner space.

The radial structure of Jin is illustrated by figure 1, shown as follows. In the illustration, the full line represents the derivation of the second major sense from the prototype; on the contrary, the dotted lines portray the extension of the minor senses in each major sense group.

We make two implications in this study. Firstly, the discrepancy between language and mental process is not one of kind, as elucidated by the functionalist [13]. From the account for Jin, we suggest that cognition underlies the meaning extension of a lexical item. On the other hand, our cognitive operation involving our bodily experiences is reflected in language. Thus, a link exists between language and other cognitive abilities. In other words, language is not a faculty independent of the other cognitive and conceptual fields. In addition, cognitive operations in perceiving the real world are encoded in language.

Secondly, the results of our study validate that meaning is built on the conceptual structure formed by the experiences of acting in the world rather than the objective reality. As evident in this research, the polysemy of Jin is a semantic network. It is not a product of an absolute, arbitrary categorization, but an outcome of a conceptualization.



Figure 1. Sense extension of Jin 
Some issues remain to be addressed relating to the sense extension of Chinese Jin. First of all, in the future studies, we wish to adopt an empirical approach to validate our analysis. In the current study, we provide an analysis for Jin on the basis of the data collected from dictionaries. Given findings about the disparity between what people actually write and say, we suggest that we should investigate naturally occurring data consulting corpora. The corpus-based approach allows us to quantify the frequency of individual metaphors and identify the central sense on the basis of such quantitative data.

Cross-linguistic comparisons should also be undertaken in future research. If it is the case that conceptual metaphors describe central processes and structures of human cognition, metaphorical mapping is not language-specific, and thus it should have explanatory power for all languages. In the cognitivist view, the most central metaphors are grounded in our human physical experience. If it is on the right track that the same conceptual metaphors exist in different languages, they tend to function in similar ways. As in the study of $\mathrm{Li}$ and Su[14], they compare and contrast Chinese Qian and Southern Min Khan from a cognitive perspective. It is found that although Chinese Qian and Southern Min Khan have common senses, they have developed distinct meaning facets. Southern Min Khan has engendered senses which Chinese Qian does not have, such as 'to endure', 'to promote', 'to stir (starch)', 'to quibble', and 'to be in a relationship with'. On the other hand, Chinese Qian has the sense 'to concern about', which Southern Min Khan does not possess. The comparison between the two lexical roots across two languages elucidates that although they have different senses, their sense expansions are undertaken in the same mechanism. Specially, the polysemous senses of Chinese Qian and Southern Min Khan are built on a network in a radical manner. We suggest that further research should be executed regarding the lexical items that denote the act 'to move forward' across languages. Take English Enter for example. Enter, just like Chinese Jin, denotes the act of moving into a closed space. However, unlike Jin, it does not encode acts such as eating, presenting something, and being upgraded to a higher state or position. Given that the two lexical items have common senses as well as distinct senses, it is worthwhile to carry out a thorough study so as to analyze the processes of sense expansion for these two morphemes. By so doing, we can explore whether people speaking different languages like Chinese and English have disparate cognitive underpinnings, and thus gain better insight into the interrelation of language and cognition.

\section{REFERENCES}

[1] G. Lakoff, M. Johnson, Metaphors We Live By, University of Chicago Press, Chicago, 1980.

[2] E. H. Rosch, Human Categorization, Advances in Cross-Cultural Psychology, Vol. 1, Academic Press, New York, 1977.

[3] M. Johnson, The Body in the Mind, University of Chicago Press, Chicago, 1987.

[4] C. Brugman, G. Lakoff, Cognitive topology and lexical networks, Lexical ambiguity resolution, Morgan Kaufmann, San Mateo, CA, 1988.

[5] M. Reddy, The Conduit Metaphor, A Case of frame conflict in our language about language, Metaphor and Thought, Cambridge University Press, Cambridge, 1993.

[6] G. Lakoff, Contemporary Theory of Metaphor, Metaphor and Thought, Cambridge University Press, Cambridge, 1993.

[7] G. Lakoff, M, Tuner, More than Cool Reason, University of Chicago Press, Chicago, 1989.

[8] W. E. Cooper, J. R. Ross, World order, Papers from the parasession on functionalism, Chicago Linguistic Society, Chicago, 1975.

[9] Ciyuan, Shangwu yin shu guan, Hunan, China, 1915.

[10] Guoyuribao Cidian, MDNA (Mandarin Daily News Association), Taipei, 1997.

[11] G. Lakoff, Women, Fire, and Dangerous Things, University of Chicago Press, Chicago, 1987.

[12] Judith A. Best. Teaching Political Theory: Meaning through Metaphor, Improving College and University Teaching, Vol. 32, No. 4, 165-168, 1984.

[13] J. I. Saeed, Semantics, Blackwell, Oxford, 1997.

[14] L. H. Li, Q. Su, A Semantic Study of Mandarin Chinese Qiān and Southern Min Khan, Chang Guan Journal of Humanities and Social Sciences, Vol. 52, No. 2, 317-351, 2012.

[15] G, Lakoff, Cognitive Semantics, Meaning and Mental Representation, Indiana U. P. Bloomington and Indianapolis, 1988. 